\title{
Apendicectomía profiláctica en la dotación Antártica: Experiencia del Hospital de la Fuerza Aérea de Chile
}

\author{
Sebastián Uribe A. ${ }^{1}$, Diego Vallejo P. ${ }^{2}$, Mauricio Jorquera ${ }^{2}$, \\ Luis Contreras ${ }^{1}$ y Felipe Celedón P. ${ }^{1}$
}

'Servicio de Cirugía Hospital de la Fuerza Aérea de Chile. Santiago, Chile.

IInterno de Medicin Universidad Mayor. Santiago,

Recibido el 11 de octubre de 2019 y aceptado para publicación el 11 de noviembre de 2019

Correspondencia a: Dr. Sebastián Uribe A. sebastian.uribea@gmail.com

\section{Prophylactic appendectomy in the Antarctic endowment: Experience of the Chilean Air Force Hospital}

\begin{abstract}
Aim: To present and characterize surgical complications of elective prophylactic appendectomies, performed in patients for the Antarctic endowment at the Hospital Clínico de la Fuerza Aérea de Chile. Materials and Method: Retrospective descriptive analysis of all patients operated of prophylactic laparoscopic appendectomy between 2013 and 2017. Demographic and surgical variables of the patients were recorded and analysed. Complications were classified according Clavien-Dindo. Results: 200 patients were included, $96 \%$ mens. Six patients (3\%) had a surgical complication, all classified as Grade I. Discussion: There are no previous reports of surgical complications on prophylactics appendectomies. The complications rate is 3\%. Conclusions: The prophylactic appendectomy is a safe surgery with a low rate of complications, although its exists and develops in a previously healthy patient.
\end{abstract}

Key words: appendectomy; prophylactic surgical procedures; postoperative complications.

\section{Resumen}

Objetivo: Reportar y caracterizar las complicaciones quirúrgicas de las apendicectomías laparoscópicas electivas profilácticas, realizadas a pacientes destinados a dotación antártica, realizadas en Hospital clínico de la Fuerza Aérea de Chile (FACh). Materiales y Método: Análisis retrospectivo descriptivo de fichas clínicas de todos los pacientes sometidos a apendicectomía laparoscópica profiláctica entre los años 2013 y 2017 en Hospital FACh. Se registraron variables demográficas y quirúrgicas de los pacientes. Las complicaciones fueron registradas y clasificadas de acuerdo a Clavien-Dindo. Resultados: Se incluyeron 200 pacientes, $96 \%$ hombres y solo $4 \%$ mujeres. Se registraron 6 pacientes $(3 \%)$ con complicaciones quirúrgicas, clasificadas como grado I según Clavien-Dindo. Discusión: No existen reportes de complicaciones en apendicectomías profilácticas. En nuestra serie éstas alcanzan el 3\%. Conclusiones: La apendicectomía profiláctica es una cirugía segura, con escasas complicaciones, pero existen y se desarrollan en un paciente que estaba previamente sano.

Palabras clave: apendicectomía; procedimientos quirúrgicos profilácticos; complicaciones quirúrgicas.

\section{Introducción}

Desde que el médico ruso Leonid Rogozov se operó a sí mismo en la base antártica de Novolázarevskaya en $1961^{1}$ y tomando en cuenta cuadros apendiculares graves en expediciones polares que han cursado incluso con mortalidad, es que hay un interés médico y gubernamental en la apendicectomía profiláctica. Pese al largo tiempo transcurrido y que varios programas antárticos la indican para su personal, no hay publicaciones en el mundo que se refieran específicamente a este procedimiento, lo que hace imposible evaluar sus riesgos y beneficios.

Por otro lado, la apendicitis aguda es una de las principales causas de abdomen agudo quirúrgico, con una prevalencia estimada de un 7\%-15\% a lo largo de la vida ${ }^{2,3}$. En los grupos de edades que habitualmente son destinados a las misiones antárticas se describe una incidencia aún mayor: de 25,5 y 43 casos/millón pacientes por año $0^{4,5}$. Se menciona que en las regiones polares esta incidencia podría verse 
aumentada, sin conocerse la causa ${ }^{6}$. Debido a esto $\mathrm{y}$ al riesgo de morbilidad potencial que incluso ha descrito mortalidad $^{7}$, la apendicectomía profiláctica se considera para ser realizadas a personas que enfrentarán condiciones de aislamiento, como lugares geográficos extremos, expediciones de navegación submarina $\mathrm{y}$, últimamente, los nuevos programas espaciales, que consideran años de confinamiento como la muy publicitada Misión a Marte.

En Chile, el programa de apendicectomía profiláctica comenzó desde la primera dotación permanente al territorio antártico, específicamente a la base "Base Aérea Antártica Presidente Eduardo Frei Montalva“ creada en 1969, posteriormente, con el establecimiento de la Base "Villa Las Estrellas" en el año 1983, se hizo extensiva al grupo familiar. Con el advenimiento de la cirugía laparoscópica, se continuó el programa con esta técnica en el año 1991. Considerando la cantidad y los años del programa, la cantidad de procedimientos gira alrededor de 1.500 pacientes intervenidos.

Dado que la apendicitis aguda se trata de uno de los diagnósticos de urgencia intraabdominales más frecuentes, existe una gran cantidad de complicaciones descritas en la literatura para la apendicectomía $^{8,9}$. Todos estos estudios están referidos a complicaciones quirúrgicas secundarias a una cirugía terapéutica por un cuadro agudo y en ellas se ve que los casos más graves tienden a tener mayor número de complicaciones como es de esperar ${ }^{10}$. Los escasos estudios de apendicectomías electivas corresponden a resecciones incidentales en relación a algún otro procedimiento quirúrgico, generalmente ginecológico $^{11-13}$.

El objetivo de este trabajo es reportar y caracterizar las complicaciones postquirúgicas de una serie de apendicectomías profilácticas laparoscópicas electivas, lo que no ha sido publicado previamente en la literatura médica.

\section{Materiales y Método}

Realizamos una revisión retrospectiva descriptiva de todos los pacientes sometidos a apendicectomía laparoscópica profiláctica en el Hospital Clínico de la Fuerza Aérea de Chile entre los años 2013 y 2017. Durante el período no hay pacientes intervenidos profilácticamente por vía abierta. Este estudio fue aprobado por el Comité de Ética. Fueron excluidos del estudio aquellos a los que se les realizó simultáneamente otro procedimiento, como colecistectomía. Dentro de las variables quirúrgicas fueron evaluados el uso de antibióticos y su duración, el tiempo operatorio, uso de bolsa para la extracción del espécimen, sutura de la aponeurosis, necesidad de conversión a apendicectomía abierta, dolor y estadía hospitalaria. Se constataron las complicaciones postquirúrgicas mediante los controles postoperatorios ambulatorios y las consultas espontáneas al servicio de urgencias y/o policlínico de cirugía en el Hospital FACh. En los casos que no había suficiente información, se contactó directamente al paciente vía telefónica o por email.

Las complicaciones fueron clasificadas de acuerdo a Clavien-Dindo ${ }^{14}$.

Si bien la técnica quirúrgica laparoscópica utilizada no se encuentra completamente estandarizada, generalmente fue como sigue: se usaron 3 trócares: T1 umbilical $12 \mathrm{~mm}$, T2 en la fosa ilíaca izquierda o suprapúbico de $5 \mathrm{~mm}$ y T3 en fosa ilíaca derecha de 5 o $12 \mathrm{~mm}$. Se usó una cámara de $10 \mathrm{~mm}$ o de $5 \mathrm{~mm}$ de diámetro, generalmente de visión frontal, pero también de $30^{\circ}$ o $25^{\circ}$. Se usó un electrocoagulador laparoscópico bipolar reusable para el meso y una ligadura prehecha desechable de ácido poliglicólico (Vycryl $^{\circledR}$ - Ethicon Surgical) o polidioxanona (PDS ${ }^{\circledR}$ Ethicon Surgical) 0 para la base apendicular. Ocasionalmente algunos cirujanos usaron, además, un clip de titanio de $10 \mathrm{~mm}$ montado en un aplicador reusable. La pieza se extrajo por T3 o T1. El uso de bolsa para la extracción de la pieza no fue de regla. El lavado peritoneal tampoco. Se suturaron los puertos de $12 \mathrm{~mm}$ y los de $5 \mathrm{~mm}$ no. Se usó profilaxis antibiótica con 1 dosis de cefazolina (Cefazolina ${ }^{\circledR}$ - Lab. Biosano) 1 g IV más metronidazol (Flagyl ${ }^{\circledR}$ - Sanofi-Aventis) 3 dosis de $500 \mathrm{mg}$ en todos los pacientes, pero en $41(20,5 \%)$ pacientes se usó tratamiento antibiótico por 5 a 7 días, de acuerdo a la indicación y preferencia del cirujano. Se realizó examen histológico a todas las piezas.

\section{Resultados}

De un total de 205 pacientes se consideraron solo 200 casos, porque en 5 se realizaron más de un procedimiento simultáneo, lo que fue un criterio de exclusión. Ninguno de estos 5 excluidos presentó alguna complicación. De los 200 pacientes incluidos, $192(96 \%)$ eran hombres. Treinta y nueve pacientes (19,5\%) presentaban una o más comorbilidades al momento de la cirugía. Diecisiete pacientes $(8,5 \%)$ presentaban una o más cirugía abdominal previa, no simultánea. Estas variables se detallan en la Tabla 1.

Ninguna cirugía requirió conversión a laparotomía. Tampoco hubo pacientes en que se perforara el apéndice como accidente intraoperatorio. Dentro 


\section{ARTíCULO ORIGINAL}

Tabla 1. Variables demográficas

\begin{tabular}{|lc|}
\hline Edad promedio & $36[28-44]$ años \\
Sexo & \\
- Hombres & $192(96 \%)$ \\
- Mujeres & $8(4 \%)$ \\
IMC & $26,9[24-29,8] \mathrm{kg} / \mathrm{m}^{2}$ \\
Cirugía abdominal previa* & $17(8,5 \%)$ \\
- Colecistectomía & $9(4,5 \%)$ \\
- Hernioplastía hiatal & $4(2 \%)$ \\
- Histerectomía & $4(2 \%)$ \\
- Cirugía bariátrica & $1(0,5 \%)$ \\
- Cesárea & $1(0,5 \%)$ \\
Comorbilidades* & $39(19,5 \%)$ \\
- Dislipidemia & $17(8,5 \%)$ \\
- HTA crónica & $12(6 \%)$ \\
- Diabetes mellitus & $9(4,5 \%)$ \\
- Hipotiroidismo & $3(1,5 \%)$ \\
\hline
\end{tabular}

*Existen pacientes con más de una variable.

Tabla 2. Complicaciones postquirúrgicas

\begin{tabular}{|lcc|}
\hline Complicaciones & $\mathbf{N}^{\mathbf{0}}$ pacientes & Clavien-Dindo \\
Infección herida operatoria & 2 & Grado I \\
Hematoma herida operatoria & 2 & Grado I \\
Dehiscencia sutura piel & 1 & Grado I \\
Fiebre de origen desconocido & 1 & Grado I \\
Total & $6(3 \%)$ & \\
\hline
\end{tabular}

de las variables quirúrgicas destacan un promedio de tiempo quirúrgico de 43,7 min (rango: 25,7$61,7 \mathrm{~min})$. El tiempo de hospitalización fue de 1 día en 137 pacientes $(68,5 \%)$, de 2 en $57(28,5 \%)$ y 3 días en los restantes $6(3 \%)$; estos últimos todos por motivos no médicos (unidad militar fuera de la región y falta de domicilio).

Se registraron 6 pacientes $(3 \%)$ que sufrieron algún tipo de complicación postoperatoria según criterios de Clavien-Dindo ${ }^{14}$, cuyo detalle se muestra en la Tabla 2. El paciente que se registra como fiebre de origen desconocido reconsultó en el Servicio de Urgencia al tercer día de alta. Fue rehospitalizado, se le realizó cultivo de la herida umbilical, hemocultivos y urocultivo, los que fueron negativos. No se usaron antibióticos y la fiebre cedió espontáneamente al día siguiente y fue dado de alta. Un paciente, ya de alta y sin signos de complicación, presentó un cuadro de gastroenteritis aguda autolimitado y se consideró como cuadro intercurrente y no directamente atribuible a la cirugía, por lo que no fue incluido en el recuento de complicaciones. Las complicaciones se describen y clasifican según los criterios de Clavien-Dindo en la Tabla 2.

El dolor postoperatorio fue evaluado y registrado durante la hospitalización con la Escala Numérica Análoga (ENA) de 0 a 10, donde se constató que $92(46 \%)$ pacientes no manifestaron sintomatología alguna, mientras que en $99(49,5 \%)$ pacientes se mantuvo un ENA $=<4$. La intensidad máxima fue ENA $7 / 10$ en un paciente.

El control ambulatorio fue en promedio a los 14 días del postoperatorio. Las complicaciones se evidenciaron tanto en los controles postoperatorios como en consultas ambulatorias espontáneas al Servicio de Urgencia. No se perdieron pacientes de la serie. El seguimiento de los pacientes en el presente estudio fue en promedio de 3 años y medio, con un seguimiento mínimo de 1 año y medio, en el caso de los pacientes operados en el periodo 2017, y hasta un seguimiento de 6 años en la serie de pacientes intervenidos en 2013.

\section{Discusión}

La importancia de la cirugía profiláctica y entre estas especialmente la apendicectomía profiláctica se ha visto incrementada en el último tiempo, tomando en cuenta el desarrollo de varios programas espaciales de larga duración por EE.UU., Rusia, Europa, Japón, India y China. Colabora a esto el hecho que buena parte de los problemas de salud que podrían producirse en estas situaciones de aislamiento y confinamiento pueden prevenirse con un tamizaje adecuado ${ }^{15}$, a diferencia de la apendicitis aguda. Llama la atención que pese a su importancia faltan datos y publicaciones que den cuenta de sus posibles riesgos y beneficios, lo que fue mencionado en la única revisión sistemática del tema ${ }^{16}$. Esta es la segunda serie presentada de pacientes a los que se les realizó una apendicectomía profiláctica en el mundo, la primera fue de complicaciones precoces (durante los primeros 5 días) en 40 pacientes, publicada como trabajo de ingreso a la Sociedad de Cirujanos de Chile por Ovalle en febrero de $1993^{17}$.

Nuestra serie muestra un $3 \%$ de complicaciones, todas leves (Clavien-Dindo grado I). Este 3\% se puede comparar favorablemente con la incidencia de complicaciones descritas para la apendicitis aguda $^{18,19}$, pero es una comparación desigual ya que es una cirugía electiva; con todo, sirve de referencia ya que no encontramos cifras sobre apendicectomía profiláctica. 
El tiempo promedio de seguimiento de los pacientes fue de un mínimo de año y medio, lo que es un tiempo suficiente para evidenciar las complicaciones postquirúrgicas. Es posible que queden fuera un número pequeño, probablemente anecdótico, de obstrucciones intestinales secundarias a la cirugía, como se menciona en la revisión sistemática de $2016^{16}$. Es necesario destacar que el único caso de obstrucción intestinal reportado en esa publicación se produjo 30 años después de una cirugía abierta. Hasta el momento, aún existe controversia en la diferencia en la frecuencia de obstrucciones intestinales posteriores a apendicectomía laparoscópica versus abierta, puesto que algunos autores destacan una menor incidencia asociada a la cirugía laparoscópica $^{20} \mathrm{y}$ otros refieren no encontrar diferencias entre ambas técnicas ${ }^{21}$. En todo caso, la serie completa de apendicectomías profilácticas de nuestro hospital que comenzó el año 1991, no registra algún paciente con esta complicación.

Un argumento en contra de esta profilaxis quirúrgica es el éxito del tratamiento no quirúrgico de la apendicitis que ha sido comunicado desde décadas atrás ${ }^{22,23}$, existiendo incluso experiencias con series comparativas aleatorizadas $^{24}$. Sin embargo, se menciona también en estas mismas experiencias que el resultado del tratamiento médico es más efectivo mientras antes se instaure, lo que en un medio de aislamiento y atención médica precaria puede que no se logre. Además, se ha descrito hasta de un $38 \%{ }^{25}$ de recidiva con este tipo de manejo.

\section{Conclusiones}

La práctica de la apendicectomía profiláctica es segura, tiene un porcentaje de complicaciones de alrededor del 3\% y que cuando se presentan son generalmente leves. Esta información puede servir -entre otras- para implementar esta profilaxis en caso de misiones en condiciones extremas o de aislamiento.

\section{Responsabilidades éticas}

Protección de personas y animales. Los autores declaran que para esta investigación no se han realizado experimentos en seres humanos ni en animales.

Confidencialidad de los datos. Los autores declaran que en este artículo no aparecen datos de pacientes.

Conflictos de interés: no hay.

\section{Bibliografía}

1. Rogozov LI. Self-operation. Soviet Antarctic Expedition Information Bulletin. American Geophysical Union. Washington DC. 1964;4:223-4.

2. Cameron J, Cameron A. Current surgical therapy. 12th. ed. Philadelphia: Elsevier; 2017;52:265-71.

3. Al-Omran M, Mamdani M, McLeod RS. Epidemiologic features of acute appendicitis in Ontario, Canada. Can J Surg. 2003;46:263-8.

4. Glover JW. The human vermiform appendix- A general surgeon's reflections Technical Journal (Creation Ex Nihilo) 1988;3:31-8.

5. Lugg DJ. Diploma in Polar Studies Thesis. University of Cambridge; Cambridge, UK: 1979. Appendicitis in polar regions.

6. Lugg DJ. Polar human biology. Chicago (IL): Year Book Medical Publishers; 1974. Antarctic epidemiology: a survey of ANARE.
7. Ball C, Kirkpatrick A, Williams D, Jones J, Polk JD, Vanderploeg J, et al. Prophylactic surgery prior to extendedduration space flight: Is the benefit worth the risk? Can J Surg. 2012;55:125-31.

8. Holzheimer R, Mannick J. Surgical Treatment: Evidence-Based and ProblemOriented. Munich: Zuckschwerdt; 2001. ISBN-10: 3-88603-714-2.

9. Gupta R, Sample C, Bamehriz F, Birch DW. Infectious complications following laparoscopic appendectomy. Can J Surg. 2006;49:397-400.

10. Álvarez R, Bustos A, Torres O, Cancino A. Apendicitis aguda en mayores de 70 años. Rev Chil Cir. 2002;54:345-9.

11. O'Hanlan KA, Fisher DT, O'Holleran MS. 257 Incidental appendectomies during total laparoscopic hysterectomy. J Soc Laparoendosc Surg. 2007;11:428-31.

12. Song JY, Yordan E, Rotman C. Incidental appendectomy during endoscopic surgery. J Soc Laparoendosc Surg. 2009;13:37683.
13. Akl MN, Magrina JF, Kho RM, Magtibay PM. Robotic appendectomy in gynaecological surgery: technique and pathological findings. Int $\mathrm{J}$ Med Robot Computer Assist Surg. 2008;4:2103.

14. Dindo D, Demartines N, Clavien PA. Classification of surgical complications: A new proposal with evaluation in a cohort of 6336 patients and results of a survey. Ann Surg. 2004;240:205-13.

15. Hamilton DR, Murray JD, Ball CG. Cardiac health for astronauts: coronary calcification scores and $\mathrm{CRP}$ as criteria for selection and retention. Aviat Space Environ Med. 2006;77:377-87.

16. David CR, Trevatt AEJ, Dixit A, Datta V. Systematic review of clinical outcomes after prophylactic surgery. Ann R Coll Surg Engl. 2016;98:1-5.

17. Ovalle C. Apendicectomía profiláctica laparoscópica. Trabajo de ingreso. Rev Chil Cir. 1993;45:42-7. 


\section{ARTÍCULO ORIGINAL}

18. Vallejos C. Apendicectomía laparoscópica $v s$ apendicectomía abierta en apendicitis aguda. Experiencia del Hospital Regional de Coyhaique. Rev Chil Cir. 2006;58:11421.

19. Rodríguez Z. Complicaciones de la apendicectomía por apendicitis aguda. Rev Cubana Cir. 2010;49(2).

Disponible en $<$ http://scielo.sld.cu/scielo. php?script=sci_arttext\&pid=S0034-

74932010000200006\&lng=es\&nrm=iso >

20. Zhang S, Du T, Jiang X, Song C.

Laparoscopic appendectomy in children with perforated appendicitis: a metaanalysis. Surg Laparosc Endosc Percutan Tech. 2017;27:262-6.

21. Mosquera M, Kadamani A, Pacheco M, Villarreal R, Ayala JC, Fajardo LP, et al. Apendicectomía laparoscópica versus abierta: comparables. Rev Colomb Cir. 2012;27:121-8.

22. Harrison PW. Appendicitis and the antibiotics. Am J Surg. 1953;85:160-3.

23. Oliak D, Yamini D, Udani VM, Lewis RJ, Amell T, Vargas H, et al. Initial nonoperative management for periappendiceal abscess. Dis Colon Rectum 2001;44:936-41.

24. Ansaloni L, Catena F, Coccolini F, Ercolani G, Gazzotti F, Pasqualini E, et al. Surgery versus conservative antibiotic treatment in acute appendicitis: A systematic review and meta-analysis of randomized controlled trials. Dig Surg. 2011;28:210-21.

25. Cobben LP, de Van Otterloo AM, Puylaert JB. Spontaneously resolving appendicitis: frequency and natural history in 60 patients. Radiology 2000;215:349-52. 